Itinéraires Itinéraires

Littérature, textes, cultures

2015-1 | 2015

Récits de société

\title{
Action-création : l'art de performance amérindien au Québec
}

Jonathan Lamy Beaupré

\section{OpenEdition}

Journals

Édition électronique

URL : http://journals.openedition.org/itineraires/2737

DOI : $10.4000 /$ itineraires.2737

ISSN : 2427-920X

Éditeur

Pléiade

Référence électronique

Jonathan Lamy Beaupré, « Action-création : I'art de performance amérindien au Québec », Itinéraires [En ligne], 2015-1 | 2015, mis en ligne le 18 décembre 2015, consulté le 20 avril 2019. URL : http:// journals.openedition.org/itineraires/2737; DOI : 10.4000/itineraires.2737

Ce document a été généré automatiquement le 20 avril 2019.

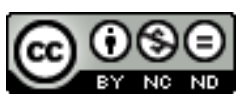

Itinéraires est mis à disposition selon les termes de la licence Creative Commons Attribution - Pas d'Utilisation Commerciale - Pas de Modification 4.0 International. 


\title{
Action-création : l'art de performance amérindien au Québec
}

\author{
Jonathan Lamy Beaupré
}

1 Les pratiques artistiques autochtones du Québec vivent depuis le début des années 2000 une véritable ébullition. Que ce soit en littérature, en arts visuels ou en cinéma, les créateurs des Premières Nations opèrent une prise de parole au sein de l'espace public et créatif, démontrant le dynamisme de leurs cultures, de même que leur singularité en tant que sujet. Ils rendent compte de la vivacité culturelle autochtone, tout en revendiquant la dimension personnelle de leurs créations. C'est ce que font Diane Robertson, Sonia Robertson, Eruoma Awashish, Louis-Karl Picard-Sioui et Mélissa Mollen Dupuis à travers leurs œuvres de performance. Celles-ci convoquent des éléments traditionnels, associés à certains rituels ou encore à la figure du Trickster ${ }^{1}$, de manière résolument actuelle et singulière, et critiquent, parfois, les stéréotypes relatifs aux Premières Nations.

\section{Performance et amérindianité}

2 En art de performance, c'est l'action - son exécution - qui constitue l'œuvre. Ce genre interdisciplinaire, parfois même indisciplinaire, met en relation « l'action dans l'art » et « l'art dans l'action », pourrait-on dire en paraphrasant le dernier titre de Richard Martel (2012). Comme l'écrit Chantal Pontbriand ([1982] 1998: 62): «La performance est davantage qu'un genre, une discipline ou une catégorie. Elle se définirait avec plus d'acuité comme un mode ou une attitude présente au sein de l'art contemporain. » Dans la performance, poursuit-elle, "c'est le questionnement du spectateur qui est recherché » (Ibid. : 63). En effet, devant une œuvre d'art-action, on se demande ce qui se passe, ce qui va se passer. La portée et la signification de l'action se construisent devant nous, et peuvent prendre différentes teintes selon le regard du spectateur.

3 Pour Dwight Conquergood (2004: 318), la performance est à la fois art, analyse et activisme, à la fois créativité, critique et citoyenneté. Dans l'ouvrage collectif The Performance Studies Reader, il écrit : 
Nous pouvons considérer la performance comme étant : 1) un travail d'imagination, un objet d'étude; 2) une pragmatique d'enquête (à la fois comme modèle et méthode), une optique ou un opérateur de recherche; 3$)$ une tactique d'intervention, un espace de lutte alternatif ${ }^{2}$. (Ibid.)

4 Les œuvres de performance sont des gestes artistiques, des actions-créations, qui ont également une dimension réflexive et engagée, portée par le corps de l'artiste. De même, les études de la performance, en plus de leur caractère analytique et critique, revendiquent une dimension créative et citoyenne, qui engage le chercheur.

Les pratiques artistiques autochtones contemporaines ne constituent pas un sujet politiquement neutre. Les étudier, en parler, peu importe les origines culturelles du chercheur, contribue nécessairement à relayer la charge critique véhiculée par ces œuvres. Bien qu'elles ne portent pas toutes à un même degré un message à caractère politique, la simple mention du travail des créateurs autochtones démontre que les Amérindiens n'ont pas «disparu», qu'ils existent toujours, au présent. Évoquer ces œuvres contribue à l'entreprise, poursuivie par nombre d'artistes eux-mêmes, consistant à prouver que leurs cultures ne doivent pas être reléguées à un passé lointain, une fable, celle du Nouveau Monde. Ainsi, les études qui abordent les enjeux contemporains autochtones participent de cet appel pour que la présence des Premières Nations soit prise en considération dans l'espace imaginaire, symbolique et politique de l'Amérique du Nord et du monde.

6 Pour James Luna ${ }^{3}$, l'un des pionniers de l'art-action autochtone, la performance permet au créateur amérindien de s'exprimer en tant que sujet tout en s'inscrivant également dans une histoire culturelle. Dans un article paru en 1991 dans lequel il décrit sa démarche, le performeur luiseno affirme :

J'ai la conviction que le travail artistique en art de performance et en installation offre une opportunité à nulle autre pareille pour les Amérindiens d'exprimer des formes d'art traditionnelles comme les cérémonies, les danses et les traditions orales à travers une pensée contemporaine, et ce, sans compromis4. (Luna $1991:$ :46)

7 Le propos de l'artiste nous invite à surmonter l'opposition entre les traditions autochtones et les modes d'expression et de création soi-disant contemporains et occidentaux (comme si peindre une toile ou écrire un poème ne pouvaient être des pratiques « authentiquement » amérindiennes). Il nous amène également à considérer les liens entre des formes d'art traditionnelles et des formes d'art performatives.

\section{Rituels actuels}

Ces liens existent chez les Premières Nations et sont documentés par l'archéologie et le patrimoine oral. Comme l'ont notamment démontré les travaux de Daniel Arsenault, plusieurs sites rupestres sont de "véritables amphithéâtres naturels» (1997: 277). Ces endroits, entre autres chez les Innus et les Inuits, n'étaient pas que des canevas de pierre, mais de petits temples, des théâtres où chants, bruitages et «jeux dramatiques de lumière » (Ibid.) étaient mis en scène de manière à la fois spectaculaire et rituelle.

9 Il n'est pas étonnant que la première œuvre de performance amérindienne produite au Québec mette en jeu cette relation de manière particulièrement vive. Elle a été réalisée par Diane Robertson (artiste de Mashteuiatsh décédée prématurément en 1993 à l'âge de 33 ans) durant le Symposium de peinture de Baie-Saint-Paul en 1986. Intitulée Kwuha pa ckan, cette œuvre consiste en une installation formée de trois tentes, un «dispositif- 
rituel », pour emprunter l'expression du commissaire et critique d'art wendat Guy Sioui Durand (1994: 20), dans lequel l'artiste a " peint le rituel de la tente tremblante » (Ibid. : 21). Pour actualiser et performer ce rituel, le mettre en art et en action, Diane Robertson a mis le feu à son installation lors de la nuit finale de l'événement. Cet acte, note encore Guy Sioui Durand, «achevait la rupture avec les symboles spécialisés du seul champ de l'art » (Ibid.). En faisant brûler son œuvre, Diane Robertson quitte le strict domaine des arts visuels pour s'inscrire dans celui de la performance, où la pratique artistique se trouve bien souvent dématérialisée. Par ce geste audacieux et radical, elle renoue également avec le rituel, adressant en quelque sorte son travail créateur aux esprits, l'offrant à la terre, plutôt qu'à un musée ou à un collectionneur.

10 S'adresser à la fois aux esprits et au monde de l'art, à la fois à l'invisible et au public présent, est une préoccupation de l'art-action autochtone. Ce faisant, l'artiste redonne en quelque sorte ce qui lui a été donné. Cette dynamique est au cœur de la démarche d'Yves Sioui Durand, fondateur de la compagnie de théâtre autochtone Ondinnok ${ }^{5}$ et réalisateur du long-métrage Mesnak ${ }^{6}$, qui a également commis quelques performances, notamment à la seizième édition du festival Polyphonix, qui s'est tenu à Québec en 19917. Signe de la volonté des organisateurs (desquels Guy Sioui Durand faisait partie) d'y inclure une présence et une parole amérindiennes, Cyrille Fontaine et Joseph McKenzie, deux chanteurs traditionnels innus, ont également participé à l'événement.

11 On doit à Guy Sioui Durand l'organisation d'événements qui ont permis la réalisation d'œuvres de performance par des artistes amérindiens, qui ont dynamisé l'art-action autochtone au Québec. Il a notamment orchestré Le Retour de l'Ours/Tortue, qui fut présenté à Montréal et au Japon en 2000 et $2001^{8}$. Yves Sioui Durand, Sylvie Paré et Sonia Robertson y ont présenté des performances. Sylvie Paré, artiste wendate qui est aujourd'hui la responsable du Jardin des Premières Nations à Montréal, y a créé $L a$ Vendeuse de paniers. Chloë Charce décrit ainsi l'action :

Portée par le rythme d'une musique qu'elle a elle-même composée, l'artiste danse dans l'obscurité en tenant quatre paniers illuminés de l'intérieur. Elle s'abandonne à une action cérémonielle du geste et du corps où elle incarne le passage d'un temps à un autre, d'un état à un autre et d'un être à un autre, en transgressant sa propre identité pour renouer avec celle d'une autre femme, inconnue et inventée par l'Autre : image stéréotypée de ce que devait avoir l'air une Indienne, avec l'artisanat comme finalité de la création autochtone. (2007 : 97)

12 Sylvie Paré conjugue ainsi une forme de métamorphisme, que l'on peut associer à la figure du Trickster, avec une critique de la représentation réductrice de l'artiste autochtone, qui ferait des paniers tressés traditionnels, et non de la performance. Elle rejoint en cela le propos du commissaire W. Richard West qui, commentant le travail de James Luna, écrit :

Les Amérindiens, autrement dit, ne servent pas à confirmer une vision contemporaine mettant à jour l'imagerie d'Edward Curtis qui tout à coup prendrait vie. Pour le dire clairement, beaucoup d'entre nous adhèrent à des traditions toujours vénérées; nous pouvons encore - certains d'entre nous - faire de magnifiques paniers et des chefs-d'œuvre perlés, mais nous sommes aussi plein de complexité contemporaine, de sensibilité sophistiquée, d'ironie et d'humour «rentre-dedans »'. (West $2005: 8$ )

13 Lors de l'événement Le Retour de l'Ours/Tortue, Sonia Robertson, artiste en arts visuels également fondatrice du festival de contes Atalukan ${ }^{10}$, incarnait pour sa part une femmecastor dans une performance intitulée Mishtuk. Après avoir dansé en évoquant la naissance d'un arbre, elle a remis des branches au public, qui a ensuite frappé sur la 
structure de bois de la longue tente, le shaputuan ${ }^{11}$. Tout comme celles de Diane Robertson et de Sylvie Paré, les performances de Sonia Robertson rejouent des principes importants de la spiritualité autochtone: le contact avec l'invisible, le passage entre le monde matériel, charnel, et celui des esprits, la transformation entre ces différents états, ainsi que le don. Ces performeuses sont les officiantes d'un rituel collectif qui engage le public, mais elles le font en convoquant des préoccupations de l'art actuel : l'éphémère, le corps comme matériau, l'échange avec le public. La frontière que l'on instaure souvent entre le traditionnel et le contemporain, l'autochtone et l'occidental, s'estompe ici.

\section{Sortir des pièges}

Lorsque l'on pense aux Premières Nations et à la performance, on pense aux danses, aux cérémonies et aux pow-wow qui ont lieu dans différentes communautés. On pense aux parures, aux plumes. Des œuvres de performance comme celles-ci nous amènent à considérer la possibilité de penser autrement l'amérindianité. Diane Robertson conjugue sa pratique rituelle avec celle de la peinture en direct. Sylvie Paré dialogue avec l'histoire de l'art; le titre et le personnage de sa performance faisant référence à une toile du peintre canadien Cornelius Krieghoff (1812-1872). Enfin, Sonia Robertson intègre des éléments de la danse butô dans sa pratique. Ces artistes créent de nouveaux métissages, rappelant que les cultures ne sont pas immuables, mais plutôt modulées et reconfigurées par des échanges. Mais elles le font surtout pour développer leur propre pratique, avec leur propre sensibilité, avec ce qui les constitue comme sujet. L'aspect culturel, comme c'est le cas pour chacun d'entre nous, n'est qu'une dimension de l'identité.

C'est là un écueil de la recherche relative à la littérature et aux arts autochtones, qui ne sont souvent considérés que pour leur autochtonicité. Comment telle toile produite par tel artiste amérindien est-elle représentative de la culture amérindienne ? Comment tel poème témoigne-t-il de l'appartenance culturelle de celui ou de celle qui l'a écrit et nous permet-il d'en faire l'expérience de façon intime? Si ces questions ne sont, bien sûr, pas dénuées d'intérêt, cette chasse à l'amérindianité, se bornant souvent à la simple reconnaissance d'éléments culturels identifiables, ne permet pas de mettre en valeur la complexité et la spécificité des œuvres et la démarche des artistes.

Jimmie Durham, possiblement le plus important artiste visuel autochtone, mais également poète, essayiste et militant, souligne que les matériaux souvent considérés comme étant traditionnellement amérindiens (les plumes, les os, le cuir) ne le sont que depuis une période très récente et ne sont certainement pas exclusifs aux Premières Nations (Durham 1992 : 143). Dans une entrevue donnée en 1993, l'artiste cherokee né en 1940 dénonçait également le fait qu'un artiste ne peut être que complètement ou pas du tout amérindien: "Soit nous ne devons pas faire référence à notre peuple et à notre situation du tout, soit nous sommes censés référer exclusivement à cela ${ }^{12} \ldots$.. (cité dans Lippard 1993 : 67). Autrement dit, selon cette perception décriée par Durham, un artiste n'est qu'autochtone ou ne l'est pas. Entre les deux, il ne semble pas y avoir de compromis possible. Comment alors analyser le travail des artistes des Premières Nations? Une telle étude tombe-t-elle invariablement dans un piège épistémologique? Commentant la production de Jimmie Durham, Jean Fisher affirme : «Il faut admettre d'emblée que les Amérindiens sont des peuples à propos desquels nous ne pouvons rien dire qui ne soit fatalement contaminé par des schémas de pensée eurocentristes ${ }^{13}$ " (1998: 101). Les artistes et les écrivains amérindiens cherchant à échapper à ces carcans, le discours sur 
leurs œuvres les y ramènent-ils fatalement? Plutôt que d'étudier l'amérindianité de manière occidentale, peut-on penser de manière amérindienne le travail des créateurs des Premières Nations? Doit-on s'amérindianiser l'esprit pour y comprendre quelque chose?

Selon l'artiste amérindienne Dolorès Contre Migwans (2008), qui se revendique d'une approche holistique, il importe de « décloisonner les pratiques et les formes artistiques». La séparation disciplinaire participerait en quelque sorte de ces "schémas de pensée eurocentristes » mentionnés par Jean Fisher. En conférence, Migwans affirmait : «nous, les Autochtones, on a naturellement une tendance à s'exprimer mais on peut pas se sentir bien quand on est juste dans une catégorie qui s'appelle "arts visuels" ou "littérature" ou "danse" ou "chant" » (Ibid.). Évidemment, il ne s'agit pas de souhaiter l'abolition pure et simple de ces catégories, mais de penser la création, de même que la démarche qui la soutient, comme un jeu de relations entre les disciplines. En effet, rares sont les créateurs autochtones qui n'œuvrent que dans un seul médium. Cette multidisciplinarité invite le chercheur à mettre en place un dialogue, qui se trouve par ailleurs au cœur de l'art de performance, entre les formes que peuvent prendre les pratiques créatrices.

\section{Une nouvelle génération}

Dans la foulée des sœurs Diane et Sonia Robertson, des frères Yves et Guy Sioui Durand, de même que Sylvie Paré, que l'on peut « ranger » dans la génération des baby-boomers, de plus jeunes artistes autochtones du Québec ont recours à la performance dans leur pratique multidisciplinaire. Les œuvres de cette nouvelle génération élargissent les frontières de la création autochtone. Soulignons d'abord que les performances se sont multipliées dans les dix dernières années, comme se sont aussi multipliés les œuvres littéraires et les films amérindiens. En 2006, alors que Sonia Robertson participait à la Rencontre internationale d'art performance de Québec, trois créateurs autochtones nés dans les années 1970 et 1980 se sont mis à œuvrer en art-action. Il s'agit Eruoma Awashish (qui pratique également la peinture et l'installation), Mélissa Mollen Dupuis (qui est aussi animatrice, conteuse et co-porte-parole d'Idle No More Québec ${ }^{14}$ ) et Louis-Karl PicardSioui (pour sa part également écrivain, artiste et commissaire). Leurs œuvres ont été montrées dans différents contextes, qui ne sont pas nécessairement et pas strictement autochtones, comme l'était par exemple Le Retour de l'Ours/Tortue. Elles sortent, d'une certaine manière, de la « réserve » de l'art amérindien. Picard-Sioui a ainsi participé à des événements comme Folie/Culture à Québec et le Festival Voix d'Amériques à Montréal, des soirées où il était le seul autochtone à se produire, alors que Mollen Dupuis a présenté des performances à la SAT (Société des arts technologiques) et au MAI (Montréal arts interculturels) lors d'activités qui n'avaient pas les enjeux autochtones comme thème principal. Si les performances des années antérieures pouvaient s'apparenter à des formes actuelles de rituels, les plus récentes véhiculent un propos beaucoup plus critique. On retrouve cette forme de mutation esthétique dans le théâtre d'Ondinnok, qui pratiquait ce que Yves Sioui Durand nomme le «théâtre de guérison », pour ensuite se consacrer à des projets qui questionnent en quoi consiste « être amérindien au xxI siècle 15 ».

19 Il se dégage de l'art-action autochtone actuel une forme de colère décolonisatrice. À l'instar des performances de James Luna (voir Lamy Beaupré 2012), on y interroge l'image conventionnelle de l'Amérindien en lien avec l'image de soi comme amérindien. Durant la 
deuxième édition de l'événement Os brûlé, à Mashteuiatsh en 2008, Eruoma Awashish a ainsi couvert son corps de miroirs (objet qui peut évoquer les relations entre colons et autochtones à une autre époque), avançant à tâtons tout en traînant des panaches de caribou qu'elle s'était attachés aux pieds. Dans cette performance intitulée La Danse du soleil, elle inverse le regard, reflétant non pas sa propre image, mais celle des spectateurs. Ici, le sujet amérindien n'est plus le support de projections fantasmatiques, liées par exemple à l'harmonie avec la nature, comme peut l'incarner avec insouciance la figure de Pocahontas, mais un miroir qui, portant son propre passé, sa propre culture, est tourné vers les autres et vers le monde. Commentant la démarche de l'artiste attikamek, Pierre Ouellet écrit dans la revue Inter :

[L]es performances et les installations rituelles d'Eruoma Awashish sont des telesmata permettant de franchir et de nous affranchir des espaces infinis, des distances secrètes qui nous séparent du monde sacré des origines et des fins, des dons premiers et des ultimes contre-dons, des données naturelles et des dettes symboliques que les offrandes, les sacrifices ou les redevances artistiques remboursent au-delà de toutes valeurs attribuables, de toutes sommes quantifiables. (2010:33)

S'inscrivant elle aussi dans cette dynamique de création comme contre-don culturel, mais sous un mode beaucoup plus ironique et frondeur, Mélissa Mollen Dupuis questionne également l'image féminine de l'amérindianité dans sa performance Totems et tabous, réalisée durant l'événement Espaces inoccupés, en 2007 à Montréal. Vêtue d'une robe qu'elle qualifiait de « rose pepto-bysmol » (Mollen Dupuis 2011), elle incarnait une squaw tout à fait kitsch, invitant le public présent à une petite promenade à travers le quartier Mile-End. Du Parc sans nom, alors occupé par la galerie Dare-Dare, l'artiste innue a guidé les participants, à la manière d'une Sacajewea postmoderne, jusqu'au Centre de rénovation L. Villeneuve. Près du mat totémique qui s'y trouve, elle invitait les personnes présentes à se faire prendre en photographie à ses côtés. Quelques-uns de ces clichés ont par la suite pris la forme de cartes postales. La performance de Mélissa Mollen Dupuis joue avec les stéréotypes, les déplace, les sabote. Elle met en pratique «une pensée amérindienne de l'art action comme outil de subversion », comme l'écrivait Guy Sioui Durand (2007 : 21) à propos de James Luna.

21 C'est aussi ce qu'a fait Louis-Karl Picard-Sioui dans As-tu du sang indien ? En 2008, alors que l'on célébrait le $400^{\mathrm{e}}$ anniversaire de la ville de Québec, le performeur et auteur wendat a personnifié un savant prononçant, sur la scène même de l'Espace $400^{\mathrm{e}}$, une allocution ironique et présentant une invraisemblable machine à mesurer la présence de sang amérindien. Au fil de son discours, le caractère raciste du personnage se dévoilait, insistant de plus en plus sur le caractère inférieur des «tribus indiennes ». Alors que le malaise grandissait dans l'audience face à ces propos, un Guerrier et une Princesse indienne (Michel Savard et Nahka Bertrand, deux artistes autochtones complices de la performance), ayant accosté en canot sur les quais du vieux port, sont venus interrompre la conférence par des chants accompagnés au tambour, entonnés par le Guerrier. Le savant a ordonné : «Appliquez l'opération Pocahontas!" Le couple fut aussitôt capturé par deux gardes (Guillaume Bastien et Guy Sioui Durand, complices eux aussi) vêtus de sarraus blancs. Le Guerrier fut neutralisé alors que l'on amenait la Princesse, ligotée, sur la scène, où une infirmière, également complice, lui fit une prise de sang. Poursuivant l'exagération théâtrale, le professeur dévoilait alors la machine à déceler le sang « impur ", " contaminé » par des gènes amérindiens. Débouchant le flacon avec ses dents, il a placé l'échantillon de sang prélevé à la Princesse sur son étrange détecteur et s'est 
dirigé vers l'auditoire pour tester les volontaires. Devant les cris de joie et mêmes les accolades que le savant offrait aux personnes dont le test était négatif, l'audience a bien compris qu'il s'agissait d'une comédie. Celle-ci s'est terminée alors que le Guerrier, s'étant libéré de ses chaînes, est venu attaquer le professeur. Guy Sioui Durand a enfin pris la parole, dissipant l'ambiguïté qui pourrait perdurer :

Vous venez de vivre avec nous l'aventure du fictif professeur Franklin et de sa fausse machine. [...] L'humour proverbial des Amérindiens peut aussi contaminer le monde de la science, le monde des recherches, de la généalogie, mais aussi nous inciter à aller voir des vraies recherches et à se demander, non pas si nous avons du sang indien, mais jusqu'à quel point nous avons $100 \%$ de sang humain ${ }^{16}$.

\section{Engager les spectateurs}

L'art-action autochtone construit ainsi des rituels collectifs où le performeur, mi-chaman mi-Trickster, engage et dérange les spectateurs. C'est ce qu'ont fait Mélissa Mollen Dupuis et Sonia Robertson lors de la cinquième édition d'Os brûlé (Lamy Beaupré 2013), à l'Université du Québec à Chicoutimi. Dans un conte performatif déluré, Mollen Dupuis a partagé avec le public un gâteau rose en forme de pénis - le sexe de Nanabozo, le fripon créateur -, étendant du crémage sur le visage de certains spectateurs. Sonia Robertson, pour sa part, circulait parmi les spectateurs, ingurgitant un très grand nombre d'hosties, de forme rectangulaire, sur lesquelles elle avait inscrit des mots en rouge tels que "peur» ou "pensionnat», en distribuant quelques-uns aux personnes présentes. L'artiste ilnue prenait sur elle et jusqu'au dégoût la violence physique et symbolique subie par les Premières Nations, dans un acte de décolonisation et de libération corporelle. Ces actions performent l'amérindianité. Elles portent ce que Judith Butler (2004) nomme l'agentivité, qui s'applique ici non pas tant à l'identité sexuelle qu'à l'identité culturelle. Ces œuvres-action nous rappellent que l'identité, si elle peut être représentée, mise en mots et en images, se crée et se récrée par des gestes posés par des sujets. Elle est un devenir, un devenir à performer. L'art-action permet de repenser ce que sont les Premières Nations, ce qu'elles étaient et ce qu'elles deviennent, mais aussi, plus généralement, ce qui compose et fonde ce qu'on appelle la culture. De plus, cette pratique invite à concevoir autrement la recherche et la création, à y inclure notamment une dimension critique et citoyenne, et peut-être également, loin de la rectitude politique qui affecte la recherche sur les questions autochtones, une certaine dose d'irrévérence.

\section{BIBLIOGRAPHIE}

Arsenault, Daniel, 1997, « Monuments, images et pratiques rituelles : vers une archéologie du paysage rituel », dans L. Turgeon, J. Létourneau et K. Fall (dir.), Les Espaces de l'identité, Québec, Presses de l'Université Laval, p. 260-279.

Butler, Judith, 2004, Le Pouvoir des mots : politique du performatif, Paris, Éditions Amsterdam.

Chamberland, Roger et Martel, Richard (dir.), 1992, Oralités / Polyphonix 16, Québec, Inter. 
Charce, Chloë, 2007, Entre-deux mondes : métissage, identité et histoire. Sur les traces de Sonia Robertson, Sylvie Paré et Rebecca Belmore, mémoire de maîtrise en histoire de l'art, Montréal, Université du Québec à Montréal.

Conquergood, Dwight, 2004, « Performance Studies: Interventions and Radical Research », dans H. Bial (dir.), The Performance Studies Reader, New York, Londres, Routledge, p. 369-380.

Contre Migwans, Dolorès, 2008, « Présentation de ma démarche dans une approche holistique, artistique et pédagogique », conférence prononcée dans le cadre de l'événement Paroles et pratiques artistiques autochtones au Québec aujourd'hui, Université du Québec à Montréal, 20 novembre.

Durham, Jimmie, 1992, [sans titre], dans D. Nemiroff, R. Houle et C. Townsend-Gault (dir.), Land Spirit Power: First Nations at the National Gallery of Canada, Ottawa, National Gallery of Canada, p. 143-147.

Durham, Jimmie et Fisher, Jean, 1998, «The Ground Has Been Covered », Art Forum, n 26, p. 99-105.

Lamy Beaupré, Jonathan, 2012, « Les détournements critiques et ironiques dans les œuvres de performance de James Luna ", dans Du stéréotype à la performance : les détournements des représentations conventionnelles des Premières Nations dans les pratiques performatives, thèse de doctorat en sémiologie, Montréal, Université du Québec à Montréal, p. 65-113.

-, 2013, «Performer avec les écureuils : Os brûlé V », Inter, art actuel, n 113, p. 50-52.

Lippard, Lucy R., 1993, " Jimmie Durham: Postmodernist “Savage” ", Art in America, vol. 81, n 2, p. 62-69.

Lowe, Truman T. et Chaat Smith, Paul, 2005, James Luna: Emendatio, Washington, Smithsonian's National Museum of the American Indian.

Luna, James, « Allow Me to Introduce Myself: The Performance Art of James Luna », Canadian Theatre Review, $\mathrm{n}^{\circ} 68$, p. 46-47.

Martel, Richard, 2012, L'Art dans l'action, l'action dans l'art (textes 2001-2012), Québec, Inter.

Mollen Dupuis, Mélissa, 2011, « Passé au futur présent », conférence prononcée dans le cadre du forum « L'oralité dans les pratiques artistiques autochtones contemporaines », Montréal, Édifice Gaston-Miron, 26 octobre 2011.

Ouellet, Pierre, 2010, « Un nouveau totémisme : rituels du souffle, de l'œil et de la marche », Inter, art actuel, $\mathrm{n}^{\circ} 106$, p. 28-35.

Pelletier, Sonia (dir.), 1994, Sur les traces de Diane Robertson (1960-1993), Montréal, PAJE Éditeur, coll. « Olive noire».

Picard-Sioui, Louis-Karl, 2008, As-tu du sang indien ?, Québec, archive vidéographique transmise par l'artiste.

Pontbriand, Chantal, [1982] 1998, « La question de la performance », dans Fragments critiques (1978-1998), Nîmes, Jacqueline Chambon, coll. « Critiques d'art », 1998, p. 61-72.

Ryan, Allan J., 1999, The Trickster Shift: Humour and Irony in Contemporary Native Art, Vancouver, Seattle, UBC Press, University of Washington Press.

Sioui Durand, Guy, « Nishk e tshitapmuk. Fable du dernier des Hurons-Wendats », dans S. Pelletier (dir.), Sur les traces de Diane Robertson (1960-1993), Montréal, PAJE Éditeur, coll. « Olive noire ", 1994, p. 18-49. 
-, 2007, « De l'art sauvage : des performeurs amérindiens dans une zone événementielle

d'envergure internationale », Inter, art actuel, $n^{\circ} 96$, p. 20-22.

Sioui Durand, Yves, Mesnak, Montréal, K-Films Amérique, 2012, 96 min.

West, W. Richard, 2005, « Lunar exploration », dans T. T. Lowe et P. Chaat Smith (dir.), James Luna: Emendatio, Washington, Smithsonian's National Museum of the American Indian, p. 7-11.

\section{NOTES}

1. Le Trickster est une figure mythologique importante des cultures amérindiennes. À la fois créateur et gaffeur, dieu et idiot, il adopte souvent la forme d'un animal et possède la capacité de se transformer et de transformer le monde, que ce soit par accident ou malice. Voir Allan J. Ryan (1999).

2. "We can think of performance (1) as a work of imagination, as an object of study; (2) as a pragmatics of inquiry (both as model and method), as an optic and operator of research; (3) as a tactics of intervention, an alternative space of struggle.» Les traductions en français de cet article sont les miennes.

3. Voir http://www.jamesluna.com.

4. «It is my feeling that artwork in the media of performance and installation offers an opportunity like no other for Indian people to express themselves in traditional art forms of ceremony, dance, oral traditions and contemporary thought, without compromise. »

5. Elle fut fondée en 1985. Voir http://www.ondinnok.org.

6. Yves Sioui Durand, Mesnak, Montréal, K-Films Amérique, 2012, 96 min.

7. Chamberland et Martel (1992). Voir également Dufour, Martel et Perrault (1993).

8. Voir http://siouidurand.org/le-retour-de-lours-tortue/.

9. "Indians, in other words, are not here to confirm some contemporary notion of updated Edward Curtis imagery come to life. To be sure, many of us still adhere to revered traditions; we can still-some of us-make beautiful baskets and beadwork masterpieces, but we are also full of contemporary complexity, sophisticated sensibility, in-your-face irony and humor. »

10. Ce festival a lieu l'été à Mashteuiatsh, réserve amérindienne située au lac Saint-Jean : http:// www.atalukan.com.

11. Pour une description plus détaillée, voir Chloë Charce (2007 : 90-92).

12. "We're either not supposed to refer to our own people and our own situation at all, or we're supposed to exclusively refer to that... »

13. "It must be admitted from the outset that Native Americans are peoples about whom we can have noting to say that is not fatally contaminated by Eurocentric patterns of thinking. »

14. Voir https://www.facebook.com/IdleNoMoreQuebec.

15. Ces expressions sont utilisées pour regrouper les différentes réalisations d'Ondinnok à travers les époques : http://www.ondinnok.org/histoire/etre-amerindien-au-21e/.

16. Louis-Karl Picard-Sioui (2008), archive vidéographique transmise par l'artiste. 


\section{RÉSUMÉS}

Les artistes amérindiens du Québec œuvrant en art de performance mettent en place des actions qui s'apparentent à des formes actuelles de rituels. Ces œuvres revêtent parfois un caractère poétique, voire "chamanique ", sont parfois plus dérangeantes, notamment à travers la charge critique quant aux clichés associés aux Premières Nations, ou encore mélangent ces deux composantes. Elles engagent autant le corps de l'artiste que le public présent, tout en faisant de l'expression culturelle amérindienne une performance à la fois artistique et identitaire.

Native artists of Quebec working in performance art set up actions that are similar to contemporary forms of rituals. These works have sometimes a poetic or "shamanic" character, are sometimes disturbing, especially through the critique of the clichés about First Nations, or mix those two components. They committed the body of the artist and the audience, while making the Native American cultural expression a both artistic and identity performance.

\section{INDEX}

Mots-clés : art de performance, art amérindien, rituel, clichés, expression culturelle

Keywords : performance art, contemporary native art, ritual, clichés, cultural expression

\section{AUTEUR}

\section{JONATHAN LAMY BEAUPRÉ}

Université Laval 